\title{
Study of systematics in anisotropy searches with AMS-02
}

\author{
K.F. Bindel, I. Gebauer*, M. Graziani and S. Zeissler \\ Institut für Experimentelle Kernphysik, Karlsruher Institut für Technologie (KIT) \\ E-mail: fabian.bindel@kit.edu, iris.gebauer@kit.edu, \\ maura.graziani@cern.ch, stefan.zeissler@kit.edu
}

The search for anisotropies in cosmic ray arrival directions with space experiments like the Alpha Magnetic Spectrometer (AMS-02) on the International Space Station is subject to a number of time and energy dependent effects originating from the detector, its environment and the data selection that need to be corrected for. Different approaches to accommodate for these effects are motivated and presented. The impact of these effects on the interpretation of anisotropy is discussed.

35th International Cosmic Ray Conference - ICRC2017

10-20 July, 2017

Bexco, Busan, Korea

*Speaker. 


\section{Introduction}

Precise measurements of the Cosmic Ray (CR) fluxes by the Alpha Magnetic Spectrometer (AMS-02) on board the International Space Station lead to unexpected features that cannot be explained within our current understanding of CR transport. Such results are the hardening in the spectra of protons, helium and lithium at a rigidity of about $300 \mathrm{GV}[1,2]$ and the excess in the positron fraction [3] which is also observed by PAMELA [4] and Fermi LAT [5]. Both results are shown in figure 1.

These features might result from astrophysical sources not yet accounted for, such as pulsars for the rise in the positron fraction and supernova remnants or Wolf-Rayet stars for the hardening of the nuclei flux spectra. Those sources need to be relatively close-by because of the energy losses experienced by electrons and positrons. Close-by sources would induce an anisotropy in the arrival direction of CRs. Thus, the angular distribution of the arrival direction of CRs is studied to provide additional information on the origin of the observed features.

To obtain a reliable measure of anisotropy, systematic effects of the experiment have to be understood. Effects in the data based on time or position dependent effects of the detector (such as its position within the geomagnetic field or its orientation towards the Sun) need to be identified, evaluated and corrected for. This work discusses how such effects are evaluated and corrected for when searching CR anisotropies.
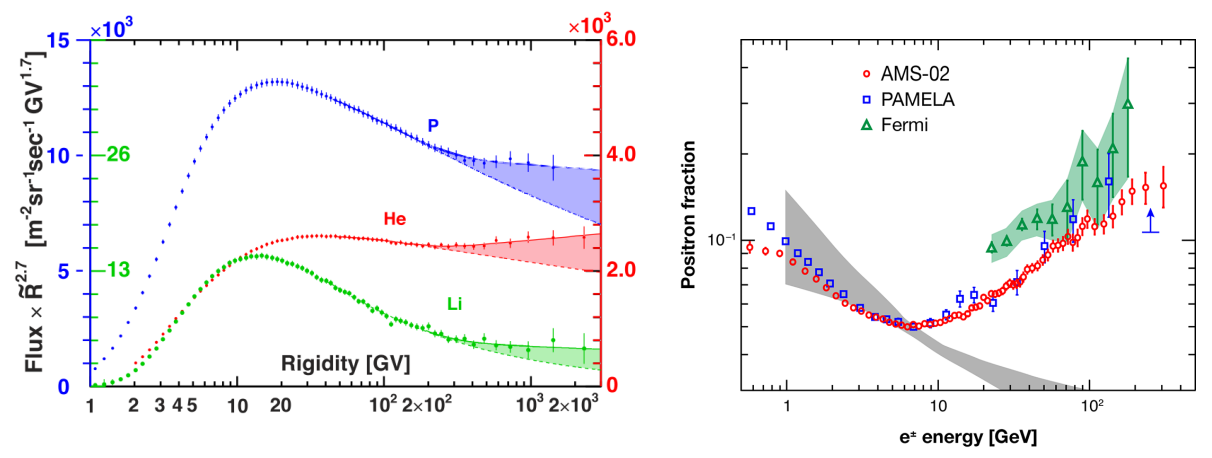

Figure 1: Left: Cosmic ray particle fluxes for protons, helium and lithium nuclei. At rigidities above $300 \mathrm{GV}$, a deviation from a single power law (dotted lines) is observed [1]. Right: Positron fraction as measured by AMS-02 [3], PAMELA [6] and Fermi LAT [5]. The gray band is the area of theoretical predictions which do not agree with the measured data above $10 \mathrm{GeV}[3,7]$.

\section{The AMS-02 detector}

Since its installation on board the International Space Station (ISS) in May 2011, AMS-02 has continually been taking data of multiple $\mathrm{CR}$ species at energies in the $\mathrm{GeV}$ to $\mathrm{TeV}$ range. The detector will continue work on board the ISS until end of operation for the space station, currently scheduled for 2024. AMS-02 is devised as an experiment with multiple subdetectors to measure different particle properties independently and in part redundantly. From top to bottom, a particle traverses a Transition Radiation Detector, a Time Of Flight detector, a Silicon Tracker, a Ring 
Imaging Cherenkov Detector and an Electromagnetic Calorimeter. A homogeneous magnetic field is created by a cylindrical permanent magnet enclosing the central part of the Silicon Tracker. A more detailed description of the detector is given in $[3,8,2]$.

\section{Anisotropy searches with AMS-02}

Anisotropy searches rely on directional information for single events. To preserve single particle information a cut-based electron and positron selection is performed. The basic quality selection follows the description in [3, 9], but no template fits in ECAL and TRD estimators are performed to identify particles. For protons, the selection follows the one presented in [8]. An overview of the $e^{+} e^{-}$and proton selection can be found in [10]. With increasing statistics, systematic effects are becoming important. To discuss the impact of systematic effects, in the following the species with the highest statistics, protons, will be used. Selected events are filled into a sky map called data map using the HEALPix ${ }^{1}$ [11] pixelation scheme. The incoming direction of each particle is projected on the celestial sphere and the map pixel value assigned to this solid angle is incremented. This is done for different coordinate systems of interest. These coordinate systems include both systems in which physical signals are expected (e.g. galactic (GAL) or geocentric solar ecliptic coordinates) as well as so-called signal-free coordinate systems, in which astrophysical signals are not expected (e. g. geomagnetic coordinates), which will be discussed in further detail below.
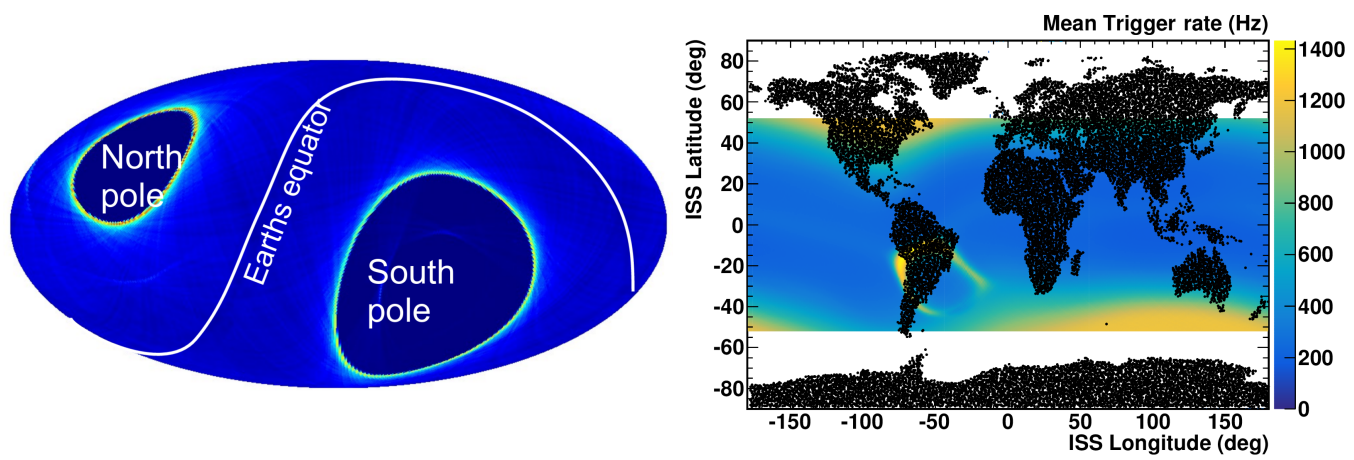

Figure 2: Left: Pointing direction of the AMS-02 detector in GAL with additional lines showing the Earth's equator and geographic poles. Right: Mean trigger rate of AMS-02. Towards the geomagnetic poles and at the South Atlantic Anomaly, the trigger rate increases, as low-energy particles hit the detector.

An isotropic sky does not look uniform to AMS-02. The left side of figure 2 shows the detector's pointing direction in GAL. The sky itself is not covered uniformly by the detector due to the ISS orbit. Additionally, due to Earth's magnetic field, the trigger rate depends on geomagnetic position, see the right side of figure 2. All selection efficiencies are, in principle, position- and therefore time-dependent. Additionally, due to aging of the detector and unstable environmental effects, some efficiencies are changing with time, giving a time-dependent origin of anisotropy, which needs to be corrected for. In order to determine the arrival directions of the incoming parti-

\footnotetext{
1http://healpix.jpl.nasa.gov/
} 
cles, a best guess for an isotropic sky map, as observed by the detector, a reference map, has to be found.

In [12], a method to create such reference maps, called IsoSkyMaps, for space-based instruments like AMS-02 is presented. Results using this method are presented as a contribution within these proceedings in [10]. An IsoSkyMap is created by getting a list of particle incoming directions $(\theta, \phi)$ in detector coordinates from the selected data events. Then, for every second of data taking, the following algorithm is applied:

1. Draw $N$ sets of incoming directions $(\theta, \phi)_{i}$ from this list

2. Calculate the arrival direction $\Psi_{i}$ from the drawn incoming directions and the detector position in the respective second in the target coordinate system

3. Weight the events with the detector livetime $T_{\text {live }}$ in this second to account for a busy trigger and a time and position dependent correction factor $\varepsilon(t, \vec{x})$, to account for time and position dependent efficiencies.

The correction factor $\varepsilon(t, \vec{x})$ is discussed below. The ratio map between data and reference map is expanded into its multipole moments, using a maximum likelihood fit method which treats bins of energy separately. A dipole $I=I_{0}(1+\delta \cos \Theta)$, where $\Theta$ is the angle towards the dipole with strength

$$
\delta=\sqrt{\rho_{F B}^{2}+\rho_{E W}^{2}+\rho_{N S}}
$$

is reconstructed with the dipole components $\rho_{\mathrm{dir}}$, is reconstructed by the fit.

Using a toy Monte Carlo approach, induced signals in GAL up to a dipole strength of $\delta=0.1$ inside the galactic plane were tested and found to be recovered with signal strengths below the statistical sensitivity of the method. The same is true for signals induced in GTOD in the East-West and Forward-Backward plane. Signals induced in NS direction in GAL are reduced by a factor of 2, while signals induced in GTOD in NS direction are fully recovered in GAL.

Since detector effects are expected to dominantly contribute along NS in GTOD and only the NS component of a possible astrophysical signal in GAL is partially recovered in GTOD, GTOD can be used as a signal-free system to correct for the detector effects discovered.

Any remaining anisotropy observed in a signal-free coordinate system is assumed to be to be of experimental origin (such as inefficiencies of the subdetectors, the position relative to Earth and the Sun) and needs to be corrected for. In this work, two different kinds of corrections are discussed, rate and efficiency corrections.

\section{Rate correction}

Above the geomagnetic cutoff, a constant rate of particles is expected. As shown in figure 2, the trigger rate of AMS-02 rises towards the geomagnetic poles, decreasing the detector's livetime due to low-energetic particles reaching the detector. Using only the livetime map as a positiondependent correction factor for the reference would be expected to flatten out any ratio map within a signal-free coordinate system by construction, possibly introducing a bias towards isotropy, removing physical signals. The normalized ratio's projection of the geomagnetic latitude is used as the correction factor $\varepsilon_{\text {rate }}$, seen on the right side of figure 3, as only the position along Earth's 


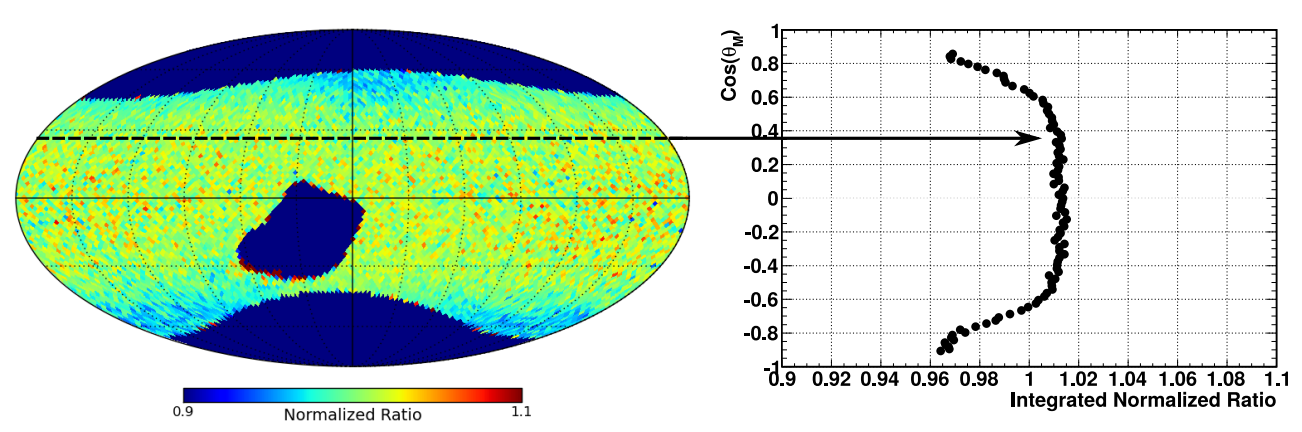

Figure 3: Rate correction. Left: Normalized ratio between data and livetime in geomagnetic coordinates for protons above $18 \mathrm{GV}$. The geographic poles as well as the South Atlantic Anomaly can be seen as points on the sky where no data is taken. At regions with a high trigger rate, i. e. close to the geomagnetic poles (cf. figure 2), the normalized ratio is lowest. Right: Normalized mean along the geomagnetic latitude. Each point is the integrated value of a single latitude ring of the ratio to the left. A drop towards the geomagnetic poles is observed.

magnetic field axis is the physical quantity responsible for the drop of detector performance. This evaluation and projection is applied for each energy bin used in the analysis and time periods of three months separately. This approach assumes no longitudinal dependence. As a cross-check of this approach, an efficiency correction is applied and described in the following.

\section{Efficiency correction}

To evaluate the efficiency of a selection cut, a control sample of the same particle type is selected by using cuts independent on the subdetector under study. To calculate the efficiency, the selected cut is applied last. This method is called tag-and-probe. The efficiency is evaluated on data, which is required to correctly take into account position and time dependent effects, which cannot be modeled by Monte Carlo simulation. An uncorrelated set of cuts with respect to the one under study is required. This is not true for studying the different subdetectors of AMS-02; correlations between the subdetectors do exist, such that each efficiency could be overestimated.

The evaluation of the efficiencies is done in geomagnetic latitude for in the energy bins of the analysis and time periods of three months. They are parameterized by $f\left(\cos \theta_{\mathrm{M}}\right)=p_{0}+$ $p_{1}\left(\cos \theta_{\mathrm{M}}\right)^{4}$, where $p_{1}$ describes the latitudinal dependence of the efficiency. This is shown in figure 4 on the left for the trigger efficiency in two different bins of energy. The IsoSkyMaps, used as reference, are created within the same time frames and energy bins. With normalizing each IsoSkyMap with the number of signal particles, a global normalization does not contribute. The single cut efficiencies can then be multiplied together and be used as a single correction function $\varepsilon_{\text {tag\&probe }}$.

The tag-and-probe approach can also be applied in two dimensions, which allows to remove possible detector effects along the geomagnetic longitude, as seen on the right side in figure 4. However, the increase in bins from one dimension to two dimensions yields a higher statistical error. As a cross-check, the two-dimensional efficiency corrections were evaluated, the results 

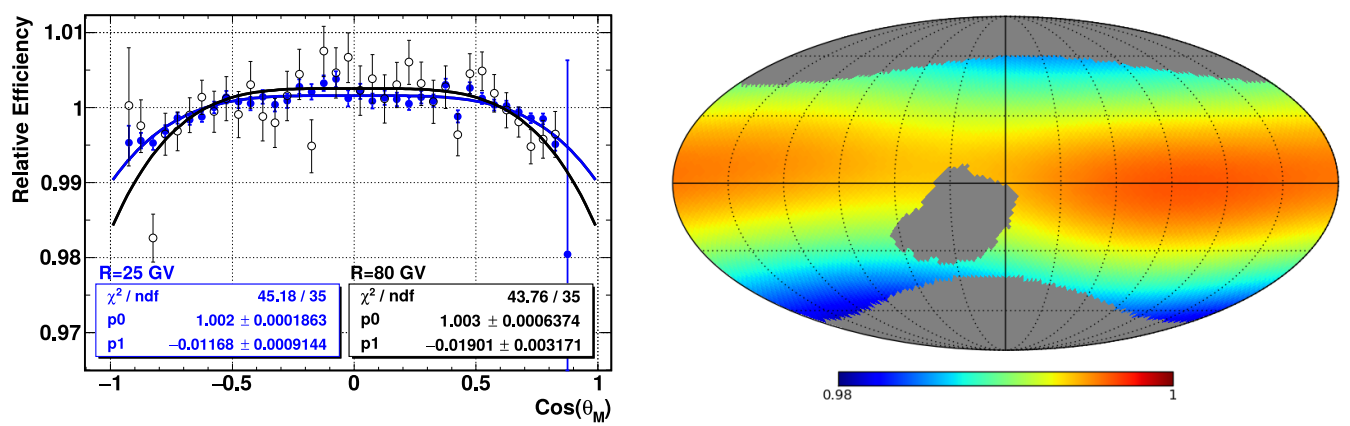

Figure 4: Left: Relative trigger efficiency for protons with rigidities of $25 \mathrm{GV}$ (blue, solid circles) and $80 \mathrm{GV}$ (black, blank circles) in geomagnetic latitude with fits following $p_{0}+p_{1}\left(\cos \theta_{\mathrm{M}}\right)^{4}$, (lines) describing the latitudinal dependence. The decrease towards the geomagnetic poles is due to the increased particle rate. Right: Two-dimensional evaluation of the TRD reconstruction efficiency for protons in geomagnetic coordinates expanded into its multipole components. A drop towards the geomagnetic poles is visible.

were consistent with the one-dimensional approach within statistical fluctuations. They can be expanded into their multipole components. Using this two-dimensional method, no bias that might remove a signal is introduced.

The assumption of no geomagnetic longitudinal dependence for $\varepsilon_{\text {rate }}$ might hide an astrophysical signal and the correction might be overestimated. $\varepsilon_{\text {tag\&probe }}$ does not have such a bias, but correlations between sub-detectors lead to a correction that is too small.

Figure 5 shows the values for $\varepsilon_{\text {tag\&probe }}$ and $\varepsilon_{\text {rate }}$ as a function of the geomagnetic latitude. A drop towards the geomagnetic poles is observed for both corrections. $\varepsilon_{\text {rate }}$ is used as the default correction for the analysis, guaranteeing more conservative retrieved dipole components from the fit.

\section{Estimating the systematic uncertainty}

The area between both one-dimensional correction approaches in geomagnetic latitude is interpreted as the systematic uncertainty of the correction, as seen in figure 5. Values within this area are randomly selected and the fit's results are re-evaluated for multiple corrections. The width of the resulting dipole component distributions constitutes the systematic error of those components in the analyzed coordinate system. Figure 5 also shows the dipole component values with both their systematic and statistical errors in galactic coordinates for protons with rigidities above $18 \mathrm{GV}$. At rigidities below $80 \mathrm{GV}$, the systematic error dominates the statistical error of the dipole component. The systematical contribution for the particular dipole components differs in different coordinate systems.

\section{Impact on dipole strength and limit}

To interpret the obtained values for the dipole strength $\delta$ from the fit, the expectation for the case of isotropy has to be known. The isotropic expectation for given statistic of $N$ particles is evaluated by filling two sky maps isotropically using Poisson distributions, one with the test 

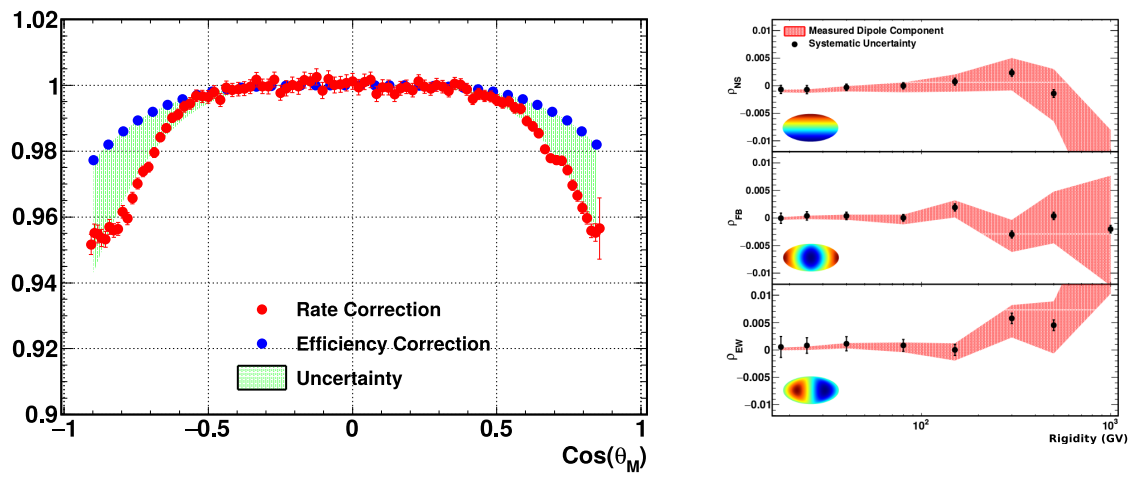

Figure 5: Left: Comparison between rate correction (red) and the one-dimensional tag-and-probe efficiency correction (blue) for protons with rigidities above $18 \mathrm{GV}$ as a function of geomagnetic latitude. The area in-between is considered as a systematic uncertainty of the analysis. Right: The systematic and statistical values and errors of the dipole components at different rigidities for protons in galactic coordinates. The dipole component reconstructed by including the systematic error is not shifted with respect to the component only affected by statistical uncertainties.

statistics of $N$ entries, one with $N_{\text {reference }} \gg N$ entries. A dipole fit is then performed for the ratio of these maps. Repeating these steps yields a distribution for $\delta$ in case of isotropy. The statistical isotropic expectation is quoted as the two-sided $68.3 \%$ interval of this distribution.

Below $80 \mathrm{GV}$ the systematic error dominates, which means that at these rigidities the the limit calculated from the dipole components is affected by systematics. The isotropic expectation needs to be increased by this offset, as seen in figure 6 .

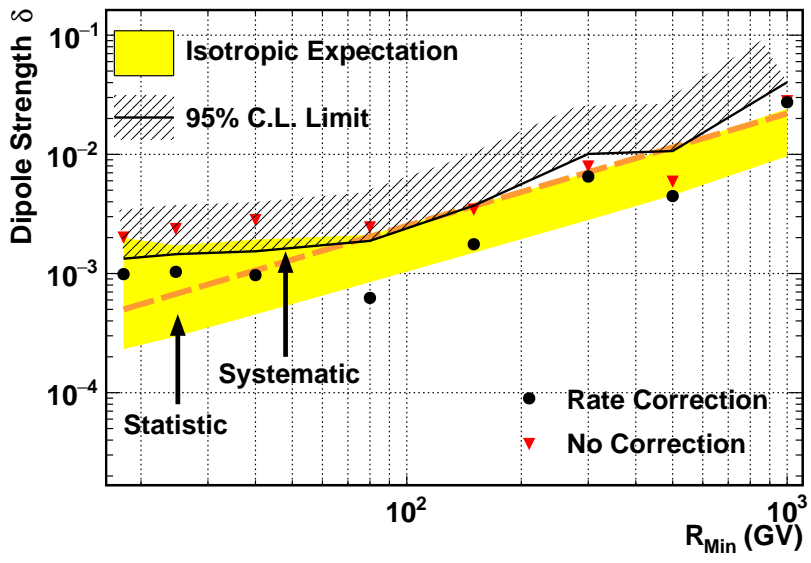

Figure 6: For protons with a reconstructed dipole in galactic coordinates, the dipole strength both with no correction (red triangles) and rate correction (black dots), as well as the isotropic expectation and the statistical limit of the fit. The systematic contribution is highlighted. The flattening out of the limit corresponds with the increased systematic uncertainty. 


\section{Summary and conclusions}

The search for dipole signals in the incoming directions of CRs a requires deep understanding of the systematic effects of the detector. Effects originating from the detector or its environment might introduce a signal and have to be understood. Using IsoSkyMaps, this can be achieved. To correct for the effects, two methods, rate and efficiency corrections, have been introduced. They are evaluated as functions along the geomagnetic latitude. The difference of these methods is used as a region of systematic uncertainty. As shown above, without these corrections, false signals for protons with rigidities below $80 \mathrm{GV}$ could be observed.

These methods are also applicable for other particle species as electrons. However, due to their reduced statistics, the systematic uncertainty does not dominate.

\section{Acknowledgments}

This work has been supported by the persons and institutions acknowledged in [2].

\section{References}

[1] S. Ting. Cosmic rays continue to confound. In: CERN Courier (Nov. 2016).

[2] M. Aguilar et al. Precision Measurement of the Helium Flux in Primary Cosmic Rays of Rigidities 1.9 GV to 3 TV with the Alpha Magnetic Spectrometer on the International Space Station. In: Phys. Rev. Lett. 115 (21 Nov. 2015), p. 211101.

[3] M. Aguilar et al. First Result from the Alpha Magnetic Spectrometer on the International Space Station: Precision Measurement of the Positron Fraction in Primary Cosmic Rays of 0.5-350 GeV. In: Phys. Rev. Lett. 110 (14 Apr. 2013), p. 141102.

[4] O. Adriani et al. An anomalous positron abundance in cosmic rays with energies 1.5-100 GeV. In: Nature 458.7238 (Apr. 2009), pp. 607-609.

[5] M. Ackermann et al. Measurement of Separate Cosmic-Ray Electron and Positron Spectra with the Fermi Large Area Telescope. In: Phys. Rev. Lett. 108 (1 Jan. 2012), p. 011103.

[6] Dmitry Malyshev, Ilias Cholis, and Joseph Gelfand. Pulsars versus dark matter interpretation of ATIC/PAMELA. In: Phys. Rev. D 80 (6 Sept. 2009), p. 063005.

[7] S. Coutu. Positrons galore. In: Physics Online Journal (6 Apr. 2013), p. 40.

[8] M. Aguilar et al. Precision Measurement of the Proton Flux in Primary Cosmic Rays from Rigidity 1 GV to 1.8 TV with the Alpha Magnetic Spectrometer on the International Space Station. In: Phys. Rev. Lett. 114 (17 Apr. 2015), p. 171103.

[9] L. Accardo et al. High Statistics Measurement of the Positron Fraction in Primary Cosmic Rays of 0.5-500 GeV with the Alpha Magnetic Spectrometer on the International Space Station. In: Phys. Rev. Lett. 113 (12 Sept. 2014), p. 121101.

[10] Iris Gebauer. Measurement of anisotropies in cosmic ray arrival directions with the Alpha Magnetic Spectrometer on the ISS. In: PoS ICRC2017 (2017).

[11] K. M. Górski et al. HEALPix: A Framework for High-Resolution Discretization and Fast Analysis of Data Distributed on the Sphere. In: apj 622 (Apr. 2005), pp. 759-771. eprint: astro-ph / 0409513.

[12] Iris Gebauer. Direction and time dependent fluxes with AMS-02. In: PoS ICRC2015 (2016), p. 404. 